\title{
Retraction Note to: Glioma grading: sensitivity, specificity, positive and negative predictive values of diffusion and perfusion imaging
}

\author{
H. R. Arvinda $\cdot$ C. Kesavadas $\cdot$ P. S. Sarma $\cdot$ \\ B. Thomas $\cdot$ V. V. Radhakrishnan $\cdot$ A. K. Gupta $\cdot$ \\ T. R. Kapilamoorthy $\cdot$ S. Nair
}

Published online: 24 July 2013

(C) Springer Science+Business Media New York 2013

\section{Retraction Note to: J Neurooncol (2009) 94:87-96 \\ DOI 10.1007/s11060-009-9807-6}

This article published in, Volume 94, Issue 1, pages 87-96, DOI 10.1007/s11060-009-9807-6, has been retracted, as it contains portions of other authors' writings on the same topic in other publications, without sufficient attribution to these earlier works being given. The principal authors of the paper acknowledged that text from background sources was mistakenly used without proper reference to the original source.

The online version of the original article can be found under doi:10.1007/s11060-009-9807-6.

H. R. Arvinda · C. Kesavadas $(\bowtie) \cdot$ B. Thomas .

A. K. Gupta · T. R. Kapilamoorthy

Department of Imaging Sciences \& Interventional Radiology,

Sree Chitra Tirunal Institute for Medical Sciences

and Technology, Trivandrum 695011, India

e-mail: chandkesav@yahoo.com

P. S. Sarma

Department of Biostatistics, Sree Chitra Tirunal Institute for

Medical Sciences and Technology, Trivandrum 695011, India

V. V. Radhakrishnan

Department of Pathology, Sree Chitra Tirunal Institute for

Medical Sciences and Technology, Trivandrum 695011, India

S. Nair

Department of Neurosurgery, Sree Chitra Tirunal Institute for

Medical Sciences and Technology, Trivandrum 695011, India 